\title{
Polarized Gravitational Waves from Gamma-Ray Bursts
}

\author{
Shiho Kobayashi ${ }^{1,2}$ and Peter Mészáros ${ }^{1,2}$ \\ 1 Center for Gravitational Wave Physics, Department of Physics, Pennsylvania State \\ University, University Park, PA 16802 \\ ${ }^{2}$ Department of Astronomy \& Astrophysics, Pennsylvania State University, University \\ Park, PA 16802
}

\begin{abstract}
Significant gravitational wave emission is expected from gamma-ray bursts arising from compact stellar mergers, and possibly also from bursts associated with fast-rotating massive stellar core collapses. These models have in common a high angular rotation rate, and observations provide evidence for jet collimation of the photon emission, with properties depending on the polar angle. Here we consider the gravitational wave emission and its polarization as a function of angle which is expected from such sources. We discuss possible correlations between the burst photon luminosity, or the delay between gravitational wave bursts and X-ray flashes, and the polarization degree of the gravitational waves.
\end{abstract}

Subject headings: gravitational waves - polarization - binaries:close - black hole physics - stars:neutron - gamma rays: bursts

\section{Introduction}

The observations of gamma-ray burst (GRB) afterglows at energies ranging from X-rays to radio have lead to an increased understanding of the possible geometry of the inferred relativistic outflow or ejecta (see van Paradijs, Kouveliotou \& Wijers 2000 for a review; also Rhoads 1999; Sari, Piran \& Halpern 1999). At least for the class of long bursts (durations $\gtrsim 10 \mathrm{~s}$ ) there is now significant evidence that the ejecta is collimated in a jet.

The evidence for jets is based on a change of the GRB light curve time-dependence power law index, and there appears to be a correlation in the sense that bursts with the largest gamma-ray fluences have the narrowest implied opening angles. Frail et al. (2001), Piran et al. (2001) and Panaitescu \& Kumar (2002) reported that the total gamma-ray energy release, after correcting for the collimation and distance determined by the afterglow observations, are narrowly clustered around $5 \times 10^{50} \mathrm{erg}$, and suggested that the broad distribution in 
fluence and luminosity for GRBs is largely the result of a wide variation of the opening angles. This result can be obtained assuming that the jet emission inside some angle $\theta_{j}$ is approximately uniform and then drops off abruptly beyond that. However, Rossi, Lazzati \& Rees (2002) and Zhang \& Mészáros (2002) showed that the observations can also be interpreted in terms of an alternative model where the jet, rather than having a uniform profile out to some definite cone angle, has a universal angle-dependent beam pattern with a luminosity per unit solid angle which is maximal along the axis, and drops off gradually away from the axis. In this model, it is the difference of viewing angles $\theta$ which causes a wide variation in the apparent luminosity of GRBs, $L \propto \theta^{-2}$.

Gravitational waves (GW) are also expected from some types of GRB (e.g. Kobayashi \& Mészáros 2002). The GW emissivity and its polarization are angle-dependent, and may in principle be measurable, depending on the signal to noise ratio and on the detector alignments. In this Letter we discuss the prospects of exploiting measurements of the gravitational polarization degree to obtain information on the nature and orientation of the GRB system, providing additional constraints on the luminosity of the bursts, and we mention possible implications for the interpretation of dark GRB and X-ray flashes.

\section{Gravitational Waves from GRB Progenitors}

Most GRB models involve a stellar system resulting in a rotating black hole and a massive $\left(\sim 0.1-1 M_{\odot}\right)$ disk of accreting matter around it. Such massive disks can form from the fall-back of debris during the formation of the central compact object, which ultimately is likely to lead to a black hole. Several scenarios could lead to a black hole-accretion disk system (e.g. Mészáros, 2002). This includes double neutron star binary mergers (Eichler et al 1989; Ruffert et al. 1997), neutron star - black hole mergers (Paczynski 1991; Janka et al. 1999) and massive stellar collapses (collapsars) (Woosley 1993; MacFadyen \& Woosley 1999).

Coalescing compact binaries are one of the most promising GW sources detectable by the laser interferometer gravitational wave observatory (LIGO), and other detectors such as VIRGO, GEO600 and TAMA300. The binary coalescence process can be divided into three phases: inspiral, merger and ringdown (e.g., Flanagan \& Hughes 1998). (1) During the inspiral phase, the gravitational radiation reaction time scale is much longer than the orbital period. As the binary losses energy by gravitational radiation, the masses gradually spiral in toward each other. (2) The merger begins when the orbital evolution is so rapid that adiabatic evolution is no longer a good approximation. The two masses then violently merge to form a black hole on a timescale comparable to a small multiple of the dynamical time. A 
bar-mode instability develops in the early stages of the merger, releasing a fraction of their rest mass energy in GWs. However, a significant fraction of the stellar material retains too much angular momentum to cross the black hole horizon promptly. This creates a temporary disk of debris material around the black hole, whose accretion can power a GRB jet. (3) The black hole is initially deformed, and in a ringdown phase radiates away the energy associated with these deformations as GWs, until it settles down into a Kerr geometry.

Massive rotating stellar collapses and their associated GW emission have been calculated numerically (e.g. Fryer et al. 1999; McFadyen \& Woosley 1999; Rampp et al 1998). These numerical estimates are not conclusive, as a number of effects (including general relativity, secular evolution, etc, see Rampp et al 1998) have been neglected, but they suggest that GW emission from massive collapses may be much less important than from compact binary mergers. On the other hand, recent semi-analytical estimates (van Putten 2001; Fryer, Holz \& Hughes 2002; Davies et al 2002) have indicated that massive collapses might emit significantly stronger GW signals than expected from the previous numerical estimates. Collapsars, i.e. massive stellar collapses leading to a GRB, require a high core rotation rate to form a centrifugally supported disk around a central, possibly spinning black hole. A high rotation rate, however, is conducive to the development of bar or fragmentation instabilities in the collapsing core or/and in the massive disk (Nakamura \& Fukugita 1989; Bonnell \& Pringle 1995; van Putten 2002). The asymmetrically infalling matter perturbs the black hole geometry, which leads to ringdown gravitational radiation.

\section{Polarization of Gravitational Waves}

We have seen that various mechanisms, including binary, bar and fragmentation instabilities and oscillations of black holes, could occur in GRB progenitor systems, resulting in GW emission. It is known that the GWs generated by a binary, bar or fragmentation instability are dominated by modes with spherical harmonic index $l=m=2$ mode (e.g. Misner, Thorne \& Wheeler 1978). The most slowly damped quasi-normal mode of a Kerr black hole also has indices $l=m=2$ (Detweiler 1980). Since this mode may be preferentially excited in the presence of binary masses or fragmentation of a massive disk, the ringdown gravitational radiation is also dominated by $l=m=2$ mode.

The polarization components of $l=m=2$ mode depend on the inclination angle $\alpha$ (e.g. Thorne 1980) as

$$
h_{+} \propto\left(1+\cos ^{2} \alpha\right), \quad h_{\times} \propto 2 \cos \alpha .
$$

GRB progenitors emit GWs more strongly along the polar axis than in the equatorial plane, the latter being the orbital plane of the binary, the disk fragments or the bar. Since the 
GRB jets are launched along the polar (angular momentum) axis (i.e. $\alpha=\theta$ ), the GRB progenitors are stronger GW sources than the average non-bursting merger or collapse, as pointed out by Kochanek and Piran (1993) in the context of a neutron star binary model. When we observe a GRB from a viewing angle $\theta$ respect to the polar axis, the GW signal is stronger by a factor of $(5 / 32)^{1 / 2}\left[(1+\cos \theta)^{4}+(1-\cos \theta)^{4}\right]^{1 / 2}$ than the value averaged over all possible viewing angles. Since for the angle dependent jet model (Rossi et al. 2002; Zhang \& Mészáros 2002) the difference of the viewing angles causes a variation of the apparent luminosity of the GRB, we expect a correlation between the apparent luminosity of GRBs and the amplitude of the associated GWs, even after distance corrections. Also in the case when the jet profile is uniform inside an opening angle $\theta_{j}$, the typical viewing angle is $\sim 2 \theta_{j} / 3$, and the correlation is still expected.

The GWs will be circular polarized when viewed along the polar axis $(\theta=0)$, while the + polarization dominates when viewed along the equatorial plane $(\theta=\pi / 2)$. Therefore, the polarization of the GWs is also correlated with the luminosity of the GRBs. We define a polarization tensor in analogy with the electromagnetic approach (e.g. Landau \& Lifshitz 1975). A plane wave at the position of a detector can be written in the form $h_{+}=\operatorname{Re}\left\{A_{+} e^{-i \omega t}\right\}$ and $h_{\times}=\operatorname{Re}\left\{A_{\times} e^{-i \omega t}\right\}$. If the plane wave is monochromatic, the complex amplitudes $A_{+}$and $A_{\times}$will be constants, whereas if the wave contains frequencies in a small interval $\Delta \omega$ we take $\omega$ to be some average frequency inside this range. $A_{+}$and $A_{\times}$are slowly varying functions of time, compared to the wave oscillation period. The polarization tensor $\rho_{a b}(a, b=+, \times)$ is defined by using the time averaged values of the amplitudes $\left\langle A_{a} A_{b}^{*}\right\rangle$ and described by the Stokes parameters $\xi_{1}, \xi_{2}$ and $\xi_{3}$ :

$$
\rho_{a b} \equiv \frac{\left\langle A_{a} A_{b}^{*}\right\rangle}{\left\langle\left|A_{+}\right|^{2}+\left|A_{\times}\right|^{2}\right\rangle}=\frac{1}{2}\left(\begin{array}{cc}
1+\xi_{3} & \xi_{1}-i \xi_{2} \\
\xi_{1}+i \xi_{2} & 1-\xi_{3}
\end{array}\right) .
$$

For the $l=m=2$ mode, the parameters are

$$
\begin{aligned}
& \xi_{1}=0, \quad \xi_{2}=\frac{8 \cos \theta\left(1+\cos ^{2} \theta\right)}{(1+\cos \theta)^{4}+(1-\cos \theta)^{4}} \\
& \xi_{3} \equiv P=\frac{2(1+\cos \theta)^{2}(1-\cos \theta)^{2}}{(1+\cos \theta)^{4}+(1-\cos \theta)^{4}}
\end{aligned}
$$

The degree of circular polarization and of linear polarization are given by $\xi_{2}$ and $\sqrt{\xi_{1}^{2}+\xi_{3}^{2}}=$ $\xi_{3} \equiv P$, respectively. Thus, GRB progenitors whose polar axes are directed towards the earth would produce brighter GRBs, and circularly polarized GWs. As the viewing angle becomes larger relative to the polar axis, the luminosity of the GRB decreases $\propto \theta^{-2}$, while the degree of linear polarization increases as $P \sim 10^{-2}(\theta / 30 \text { degree })^{4}$. 


\section{Polarization Measurement}

The response of an interferometer (interferometer 1 ) to the gravitational radiation is given by a linear combination of two polarization components $m_{1}=F_{+, 1} h_{+}+F_{\times, 1} h_{\times}$where the antenna patterns $F_{+, 1}$ and $F_{\times, 1}$ depend on the orientation of the interferometer with respect to the GW source (e.g. Finn \& Chernoff 1993). We assume that the interferometer arms are of the same length and that they meet at right angles. Define a right-handed coordinate system with one interferometer arm along the $x$-axis and the other along the $y$-axis. For simplicity, we assume that the source is in the direction of the $z$-axis. Since we can determine the position of a source in the sky by using observations of the GRB and afterglow, it is possible to generalize the following discussion to the case of a source with an arbitrary sky position. The angular momentum vector of the source (direction of the GRB jet) may be oriented in an arbitrary direction. We assume that the projection of the angular momentum vector to the $x-y$ plane makes an angle $\zeta$ with the $x$-axis. With these conventions, the antenna patterns are given by $F_{+, 1}=\cos 2 \zeta$ and $F_{\times, 1}=\sin 2 \zeta$.

To determine the polarization of GWs, one needs a network consisting of at least two interferometers which have different (i.e. non-parallel) arm orientations. Consider an identical interferometer (interferometer 2) at the same location as interferometer 1 . (If we set the arrival time of the GRB signal as the origin of time at each interferometer, we can correct for the actual physical separation so that the two interferometers can be always considered to be at the same location). We assume that the interferometer 2 is rotated by an angle $-\pi / 4$ around the $z$-axis with respect to the interferometer 1 . The response of the interferometer 2 is $m_{2}=F_{+, 2} h_{+}+F_{\times, 2} h_{\times}$, and the antenna patters are given by $F_{+, 2}=-\sin 2 \zeta$, and $F_{\times, 2}=\cos 2 \zeta$.

The detection of the polarization of GWs requires observations with a high signal-tonoise ratio $(\mathrm{SNR}) \rho$. A detection is likelier in an optimal case where the wave forms of the polarized components, $f_{+}$and $f_{\times}$, are known. We define the noise-weighted inner product as

$$
\left\langle s_{i}, f_{a}\right\rangle=4 \operatorname{Re} \int_{0}^{\infty} \frac{\tilde{s}_{i}(f) \tilde{f}_{a}^{*}(f)}{S_{h}(f)} d f
$$

where $\tilde{s}_{i}(f)$ denotes the Fourier transform of the outputs of the two interferometers $s_{i}(t)=$ $m_{i}(t)+n_{i}(t)(i=1,2), n_{i}(t)$ is the noise of the interferometers, $S_{h}(f)$ is the one-sided noise power spectral density and $\tilde{f}_{a}$ is the Fourier transform of $f_{a}(a=+, \times)$. We normalize the functions $f_{a}$ as $\left\langle f_{a}, f_{b}\right\rangle=\delta_{a b}$, hence the dispersion of the noise is unity $\sigma^{2}=\overline{\left\langle n_{i}, f_{a}\right\rangle^{2}}=1$ where the overline represents ensemble average. The linear polarization degree $P=\xi_{3}$ is

$$
P=\frac{\left\langle m_{1}, f_{+}\right\rangle^{2}+\left\langle m_{2}, f_{+}\right\rangle^{2}-\left\langle m_{1}, f_{\times}\right\rangle^{2}-\left\langle m_{2}, f_{\times}\right\rangle^{2}}{\left\langle m_{1}, f_{+}\right\rangle^{2}+\left\langle m_{2}, f_{+}\right\rangle^{2}+\left\langle m_{1}, f_{\times}\right\rangle^{2}+\left\langle m_{2}, f_{\times}\right\rangle^{2}} .
$$


Since the outputs of the interferometers $s_{i}$ are always superpositions of signals $m_{i}$ and noises $n_{i}$, the inner products $\left\langle m_{i}, f_{a}\right\rangle$ themselves are not observable. We estimate $\left\langle m_{i}, f_{a}\right\rangle$ by $\left\langle m_{i}, f_{a}\right\rangle \sim\left\langle s_{i}, f_{a}\right\rangle$. Substituting this into eq. (6), we can get an estimate $P^{\prime}$ of the polarization degree. This approximation introduces an error $\Delta$ in the estimate of the polarization degree $P$, given by

$$
\begin{aligned}
\Delta & \equiv \frac{P^{\prime}-P}{P}=\frac{1+(A-B) / P}{1+A+B}-1, \\
A & =2 a_{+}\left(\lambda_{1+} \cos 2 \zeta-\lambda_{2+} \sin 2 \zeta\right)+\lambda_{1+}^{2}+\lambda_{2+}^{2}, \\
B & =2 a_{\times}\left(\lambda_{1 \times} \sin 2 \zeta+\lambda_{2 \times} \cos 2 \zeta\right)+\lambda_{1 \times}^{2}+\lambda_{2 \times}^{2},
\end{aligned}
$$

where $a_{+}=\sqrt{2}\left(1+\cos ^{2} \theta\right)\left[(1+\cos \theta)^{4}+(1-\cos \theta)^{4}\right]^{-1 / 2}, a_{\times}=2 \sqrt{2} \cos \theta\left[(1+\cos \theta)^{4}+(1-\right.$ $\left.\cos \theta)^{4}\right]^{-1 / 2}, \lambda_{i a}=\left\langle n_{i}, f_{a}\right\rangle / \rho$ and the SNR $\rho$ is $\rho^{2}=\left\langle h_{+}, f_{+}\right\rangle^{2}+\left\langle h_{\times}, f_{+}\right\rangle^{2}$. The fluctuations $\lambda_{i a}$ are of order of $\rho^{-1}$. Thus, a SNR of $\rho>P^{-1}$ is required, if one wants to determine the polarization degree to an accuracy of order $P$.

To evaluate the error $\Delta$ numerically, we assume that the $\lambda_{i a}$ are normally distributed, and that the projection of the angular momentum on the orbital plane $\zeta$ relative to the $x$-axis is uniformly distributed between 0 and $2 \pi$. Figure 1 shows the distributions of $\Delta$ for $10^{6}$ random realizations in the case of a $1 \%$ polarization degree $\left(P=10^{-2}\right)$. The SNR was assumed to be $\rho=100,300,500$ or 1000 , for which the fractions of the realizations with large errors $(|\Delta|>0.5)$ are $80 \%, 44 \%, 20 \%$ and $1 \%$, respectively. Therefore, $\rho \sim 10 P^{-1}=1000$ is required to determine a $1 \%$ polarization degree.

The LIGO interferometers are coaligned, and thus cannot by themselves determine the polarization degree of GWs, although a network consisting of LIGO and other interferometers could in principle do it. We assume here an interferometer identical to the LIGO detectors with an optimal orientation (rotated by $\pi / 4$ with respect to the LIGO interferometers). If some fraction of GRB progenitors are double neutron star mergers, the SNR is given by $\rho_{\text {ave }} \sim 16(d / 100 \mathrm{Mpc})^{-1}$ (e.g. eq. (16) in Kobayashi \& Mészáros 2002) where $d$ is the distance to the binary. This estimate was obtained from an average over different possible orientation of the source and interferometer. The SNR $\rho$ is larger by factor of $(5 / 4)\left[(1+\cos \theta)^{4}+(1-\cos \theta)^{4}\right]^{1 / 2}$. Then, in an optimal case, we can determine a $1 \%$ polarization degree up to $d_{\max } \sim 7 \mathrm{Mpc}$. If we assume that $\sim 1000$ GRBs happen in a year within $\sim 3000 \mathrm{Mpc}$, the closest event in a year is, on average, at $d \sim 300 \mathrm{Mpc}$. Since the position in the sky is random, the SNR is smaller by a factor of $\sqrt{2 / 5}$ than in the direction of the z-axis. The LIGO is most sensitive around $f_{0} \sim 150 \mathrm{~Hz}$ and the sensitivity is about $\sqrt{f_{0} S_{h}\left(f_{0}\right)} \sim 3 \times 10^{-23}$. Since $d_{\max }$ is proportional to $S_{h}^{-1 / 2}$, a future detector with sensitivity $\sqrt{f_{0} S_{h}\left(f_{0}\right)} \sim 4 \times 10^{-25}$ would enable us to measure a polarization degree of $1 \%$ in a timescale of one year. 
Frail et al. (2001) show that the observed distribution of jet opening angles (or viewing angles for the angle dependent jet model) is $f_{o b s} \propto \theta_{j}^{-3.5}\left(\theta_{j}>0.05\right)$. Assuming this result, the probability that GWs from a GRB have a polarization degree larger than $P=10^{-2}$ is given by $\sim 3 \times 10^{-2}\left(P / 10^{-2}\right)^{-0.4}$. However, the $f_{\text {obs }}$ are estimated from observations of typical GRBs at redshifts $z \sim 1$, and one might fail to detect a significant fraction of the $\operatorname{dim}$ GRBs at large viewing angles. Therefore, when we study GWs from nearby GRBs, the probability that one observes GWs with a large polarization degree could be much larger than the above estimate.

\section{Discussion and Conclusions}

We have argued that GRB progenitors are likely to emit $l=m=2$ gravitational waves $(\mathrm{GWs})$, which are circularly polarized on the polar axis, while the + polarization dominates on the equatorial plane. Recent GRB studies suggest that the wide variation in the apparent luminosity of GRBs are caused by differences in the viewing angle, or possibly also in the jet opening angle. Since GRB jets are launched along the polar axis of GRB progenitors, correlations among the apparent luminosity of GRBs and the amplitude as well as the polarization degree of the GWs are expected.

At a viewing angle larger than the jet opening angle $\theta_{j}$ (which may be defined also in the case of a universal angle-dependent jet profile) the GRB $\gamma$-ray emission may not be detected. However, in such cases an "orphan" long-wavelength afterglow could be observed, which would be preceded by a pulse of GWs with a significant linearly polarized component. An expanding jet with an opening angle $\theta_{j}$ behaves, as long as its Lorentz factor $\gamma>\theta_{j}^{-1}$, as if it were part of a spherical shell, but relativistic beaming effect allows only observers at viewing angles $<\theta_{j}$ to observe the emission from the jet. (By contrast, since $\mathrm{GW}$ emission is expected from the central engine itself, GWs are not subject to such extreme beaming). As the jet slows down and reaches $\gamma \sim \theta_{j}^{-1}$, the jet begins to expand laterally, and its electromagnetic radiation begins to be observable over increasingly wider viewing angles. Since the opening angle increases as $\sim \gamma^{-1} \propto t^{1 / 2}$ (Sari et al 1999), at a viewing angle $\theta>\theta_{j}$, the orphan afterglow begins to be observed (or peaks) at a time $t_{p} \propto \theta^{2}$ after the detection of the GW burst. The polarization degree and the peak time should be correlated as $P \propto t_{p}^{2}$.

A new type of fast transient source, called "X-ray flashes", have recently been observed with the BeppoSAX satellite (Kippen et al. 2002). Apart from their large fraction of X-rays $(\sim 2-10 \mathrm{keV})$, the overall properties of these events are similar to those of GRBs. Recently, Yamazaki, Ioka and Nakamura (2002) and Woosley, Zhang \& Heger (2002) suggested that 
these events may be GRBs with large viewing angles. If this is the case, linearly polarized GWs should be observed prior to the X-ray flashes. The degree of polarization should be positively correlated with longer delays and with the softness of the X-ray flashes, which increase with angle.

Since the degrees of linear and circular polarization depend on the viewing angle, a determination of the polarization degree would be a measure of the viewing angle. Such measurements, which are likely to require the advent of a future generation of detectors, could provide a new tool for estimating the absolute luminosity of GRBs, including its photon component. By comparing the estimated absolute photon luminosity with the apparent luminosity, the distance to the source may be estimated independently of any redshift measurement. No optical afterglows have been found for about half of all the GRBs detected by BeppoSAX (the so called "dark GRBs"), and the present method would have the potential to help determine or constrain the distances to such dark GRBs.

We thank L. S. Finn, B. Owen, I. Jones and P. Sutton for useful discussions. We acknowledge support through the Center for Gravitational Wave Physics, which is funded by NSF under cooperative agreement PHY 01-14375, and through NSF AST0098416 and NASA NAG5-9192.

\section{References}

Bonnell, I.A. \& Pringle, J.E. 1995, MNRAS, 273, L12.

Davies, M.B., King, A.,Rosswog,S. \& Wynn,G. 2002, ApJ, 579, L63.

Detweiler,S. 1980, ApJ, 239, 292.

Eichler, D., Livio,M., Piran,T. \& Schramm,D.N. 1989, Nature, 340, 126.

Finn, L.S. \& Chernoff,D.F. 1993, Phys. Rev. D, 47, 2198.

Flanagan,E.E. \& Hughes, S.A. 1998, Phys. Rev. D, 57, 4535.

Frail,D.A. et al. 2001, ApJ, 562, L55.

Fryer, C.L., Woosley,S.E., Herant,M. \& Davies,M.B. 1999, ApJ, 520, 650.

Fryer, C.L., Holz, D.E. \& Hughes,S.A. 2002, ApJ, 565, 430.

Janka,H.T., Eberl,T., Ruffert,M. \& Fryer,C. 1999, ApJ, 527, L39.

Kippen, R.M., Woods,P.M., Heise,J., in’tZand,J.J.M., Briggs,M.S. \& Preece,R.D. 2002, preprint (astro-ph/0203114).

Kobayashi,S \& Mészáros,P. 2002, submitted to ApJ, astro-ph/0210211.

Kochanek,C. \& Piran, T. 1993, ApJ, 417, L17.

Landau,L.D. \& Lifshitz,E.M. 1975, The Classical Theory of Fields, (New York, Pergamon). MacFadyen,A.I. \& Woosley, S.E. 1999, ApJ, 524, 262.

Mészáros, P. 2002, Annu. Rev. Astron. Astrophys., 40,137.

Misner,C.W., Thorne,K.S. \& Wheeler,J.A., Gravitation (Freeman, San Francisco 1973). 
Nakamura,T. \& Fukugita,M. 1989, ApJ, 337, 466.

Rampp,M., Muller,E. \& Ruffert,M. 1998, A\&A, 332, 969.

Rossi,E., Lazzati,D. \& Rees,M.J. 2002, MNRAS, 332, 945.

Paczynski,B. 1991, Acta Astronomica, 41, 257.

Panaitescu,P. \& Kumar,P. 2002, ApJ, 571, 779.

Piran,T., Kumar,P., Panaitescu,A. \& Piro,L. 2001, ApJ, 560, L167.

Rhoads,J.E. 1999, ApJ, 525, 737.

Ruffert,M., Janka,H.T., Takahashi,K. \& Schaefer,G 1997, A\&A, 319, 122.

Sari,R., Piran,T.\& Halpern,J.P. 1999, ApJ, 519, L17.

Thorne,K.S. 1980, Rev. Mod. Phys., 52, 299.

Yamazaki, R., Ioka,K. \& Nakamura,T. 2002, ApJ, 571, L31.

van Paradijs,J., Kouveliotou,C. \& Wijers,R.A.M.J. 2000, Annu. Rev. Astron. Astrophys., 38,379 .

van Putten, M.H.P.M. 2001, ApJ Lett., 562, L51.

van Putten, M.H.P.M. 2002 , ApJ Lett., 575, L71.

Woosley,S. 1993, ApJ, 405, 273.

Woosley,S. Zhang,W \& Heger,A. 2002 , preprint (astro-ph/0206004).

Zhang,B. \& Mészáros,P. 2002, ApJ, 571, 876. 


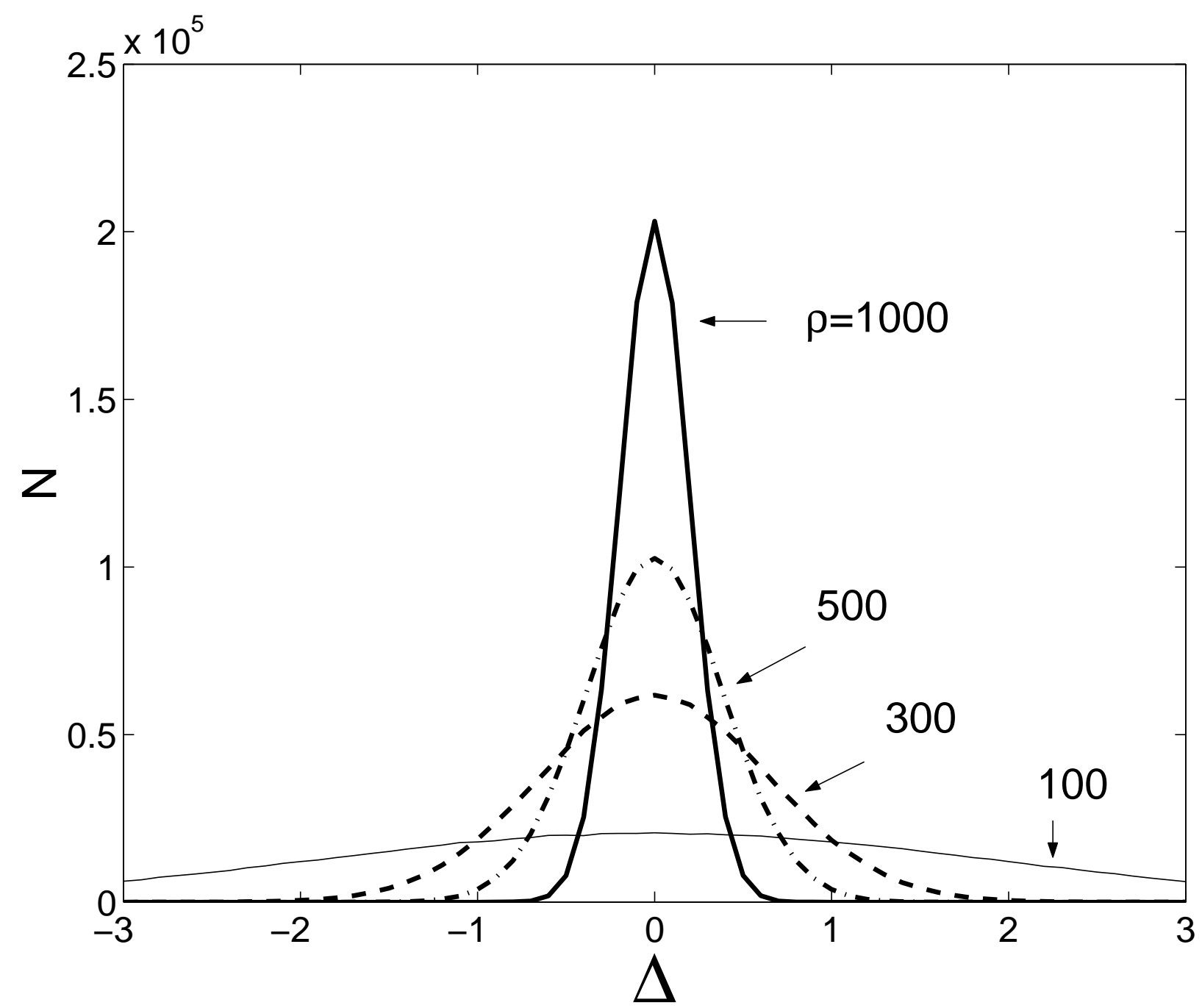

Fig. 1.- Distribution of errors $\Delta$ for a polarization degree $P=10^{-2}$, signal to noise ratios $\rho=100$ (thin solid), 300 (dashed), 500 (dashed dotted) and 1000 (thick solid), based on $10^{6}$ random realizations. The size of the bins is 0.1 . 